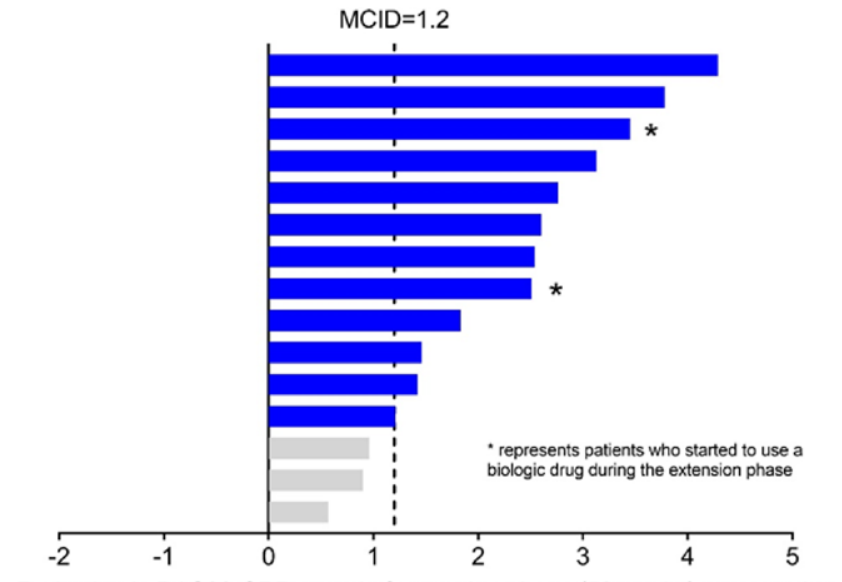

Reduction in DAS28-CRP at end of extension phase (52 weeks) compared to the study baseline

Disclosure of Interests: Sara Marsal Speakers bureau: BMS, Pfizer, UCB, Celgene, Roche, Sanofi, Consultant of: Pfizer, Abbvie, Roche, Celgene, Galapagos, MSD, UCB, BMS, Sanofi, Grant/research support from: Pfizer, Abbvie, Roche, Celgene, MSD, UCB, BMS, Novartis, Janssen, Sanofi, Héctor Corominas: None declared, Juan Jose de Agustin: None declared, Carolina Perez-Garcia: None declared, Maria Lopez Lasanta: None declared, Helena Borrell Paños: None declared, D Reina-Sanz: None declared, Raimón Sanmartí: None declared, J. Narváez: None declared, Clara Franco-Jarava: None declared, Charles Peterfy Speakers bureau: Novartis, Bristol Myers Squibb, Amgen, Consultant of: Multiple companies on behalf of Spire Sciences Inc., Jose Antonio Narvaez: None declared, Vivek Sharma Shareholder of: Nēsos Corp, Employee of: Nēsos Corp, Konstantinos Alataris Shareholder of: Nēsos Corp, Employee of: Nēsos Corp, Mark C. Genovese Shareholder of: Gilead Sciences, Nēsos Corp, Employee of: Gilead Sciences, Matthew Baker Shareholder of: Nēsos Corp, Consultant of: Nēsos Corp DOI: 10.1136/annrheumdis-2021-eular.2628

\section{AB0265 OPIOIDS AND ANALGESIC USE IN EARLY RHEUMATOID ARTHRITIS: A LONGITUDINAL ANALYSIS OF LINKED REAL-WORLD PRESCRIPTION DATA}

M. H. Derakhshan ${ }^{1}$, F. Morton ${ }^{1}$, G. E. Fragoulis ${ }^{2}$, C. Paterson ${ }^{1}$, J. Dale ${ }^{3}$ N. Basu', I. Mcinnes ${ }^{1}$, D. Porter ${ }^{4}$, S. Siebert ${ }^{1}$ on behalf of Scottish Early Rheumatoid Arthritis (SERA) Investigators. ${ }^{1}$ University of Glasgow, Institute of Infection, Immunity and Inflammation, Glasgow, United Kingdom; ${ }^{2}$ University of Athens, Laiko General Hospital, Athens, Greece; ${ }^{3} \mathrm{NHS}$ Lanarkshire, University Hospital Hairmyres, Glasgow, United Kingdom; ${ }^{4} \mathrm{NHS}$ Greater Glasgow and Clyde, Gartnavel General Hospital, Glasgow, United Kingdom

Background: Large numbers of patients with rheumatoid arthritis (RA) receive regular opioids despite significant toxicity and a lack of evidence supporting their use in non-cancer pain. In order to address this situation, we need to understand when opioids are started in early RA where this has not been studied.

Objectives: To examine the temporal trend of opioid prescriptions before and after RA symptom onset and to compare this with DMARD and NSAID prescriptions.

Methods: RA participants (cases) were recruited as part of the Scottish Early Rheumatoid Arthritis (SERA) inception cohort ${ }^{1}$. Controls without RA (five per case), matched for sex, age and post code over the same time period, were obtained through routine data linkage. Prescription data between Jan 2009 to Nov 2019 of cases and matched controls were compared using date of RA symptom onset as reference point. The Prescriptions Per Participant (PPP) for each three-month block was estimated by dividing the number of prescribed drugs in the selected drug classes (assigned using the British National Formulary) in that time block by the number of participants in each group. The differences between mean PPP of the RA cases and controls in each time block were tested by t-test for independent groups and subsequent adjustment for multiple testing.

Results: $1,720,335$ prescriptions were available for analysis with 421,961 items for 950 RA cases and 1,299,374 items for 4,558 matched controls. As expected,
DMARD prescriptions in the SERA cases increased after the symptom onset period and were then sustained (Figure 1: top left panel). NSAID prescriptions in RA cases peaked during the 3 months after symptom onset and then reduced progressively (top right panel). Opioid analgesic prescriptions for the RA cases increased two-fold during the reference period and then reduced 6-9 months post-symptom onset. However, unlike NSAIDs, after this there was no further significant reduction in opioid prescriptions in the RA cases, which remained stable and significantly higher than in the controls for the remaining study period. The non-opioid analgesic mean PPP increased sharply at the time of symptom onset, with a steady gradual upward trend over time (lower right panel).
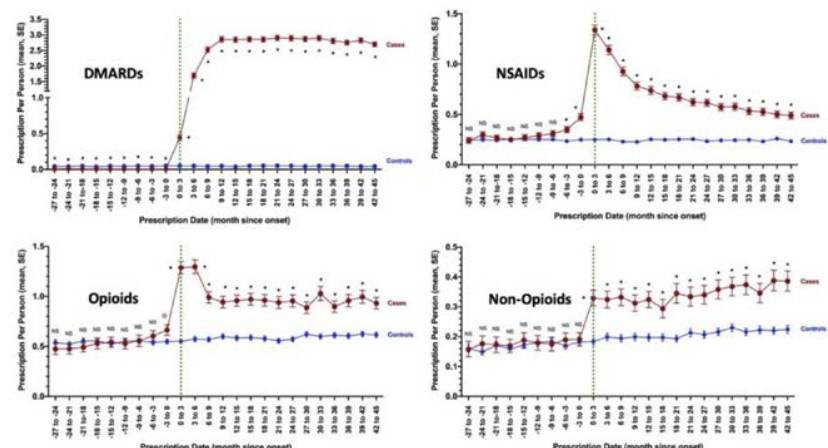

Conclusion: Opioid prescriptions increase significantly at the time of RA symptoms onset. Despite rapid introduction of DMARDs and resultant reductions in NSAIDs, analgesic use remains significantly higher than in controls. Further research is required to identify the factors associated with persistent opioid use in early RA with interventions aimed at the first 6 months. REFERENCES:

[1] Dale et al. BMC Musculoskelet Disord. 2016;17:461.

Acknowledgements: The work was supported by Health Data Research UK which receives its funding from HDR UK Ltd funded by the UKRI MRC, EPSRC, Economic and Social Research Council, Department of Health and Social Care (England), Chief Scientists Office of the Scottish Government Health and Social Care Directorates, Health and Social Care Research and Development Division (Welsh Government), Public Health Agency (Northern Ireland), British Heart Foundation (BHF) and the Wellcome Trust.

The SERA study was jointly funded by the Chief Scientists Office Scotland and Pfizer Ltd.

Disclosure of Interests: None declared

DOI: 10.1136/annrheumdis-2021-eular.2672

\section{AB0266 METHOTREXATE AND CARDIOVASCULAR RISK IN RHEUMATIC DISEASES:A COMPREHENSIVE REVIEW}

F. Verhoeven ${ }^{1,2}$, C. Prati ${ }^{1,2}$, M. Chouk ${ }^{1}$, C. Demougeot ${ }^{2}$, D. Wendling ${ }^{1,3} \cdot{ }^{1} \mathrm{CHRU}$ Besancon, Rheumatology, Besançon, France; ${ }^{2}$ Université de Bourgogne - Franche Comté, EA 4267 PEPITE, Besançon, France; ${ }^{3}$ Université de Bourgogne - Franche Comté, EA 4266 EPILAB, Besançon, France

Background: The management of inflammatory rheumatic disease has evolved in the last decade with the importance of the management of comorbidities. Methotrexate is the cornerstone of inflammatory rheumatic disease management, but its cardiovascular effects are still poorly understood

Objectives: To assess the cardiovascular impact of methotrexate in inflammatory rheumatic disease.

Methods: A systematic review of the literature, following the prisma recommandations, was performed on the PubMed and Embase databases with the following keywords: ("Methotrexate") AND ("cardiovascular"). We included papers written in English and including patients older than 18 years.

Results: 570 references were identified and, 36 articles were kept for analysis. The mechanism of action of methotrexate lies mainly on the antagonism of purines. It reduces systemic inflammation, oxidative stress.

In Rheumatoid arthritis, the use of methotrexate was associated with a decreased incidence of high blood pressure, an improvement of the lipid profile and of the insulin resistance. Major adverse cardiovascular events were decreased with methotrexate. The effects of methotrexate on the endothelial 